\title{
Articles of Significant Interest in This Issue
}

\author{
Mediation Analysis Shows that the Amount of Dietary Fiber Consumed during \\ Weaning Influences Gut Microbiota Development \\ The introduction of complementary foods to the milk diet of early infancy affects the \\ development of the gut microbiota. Leong and colleagues (e00914-18) aimed to \\ determine whether encouraging early introduction of an adult-type diet results in \\ alterations to the gut microbiota compared to traditional spoon-feeding during wean- \\ ing. Evaluation of dietary intake and fecal microbiota compositions by mediation \\ analysis showed that the greater complexity of the microbiota of spoon-fed infants at \\ 12 months of age was associated with a higher intake of dietary fiber (fruit and \\ vegetables). Mediation modeling is commonly used in health research but not in \\ microbiota studies of the developing infant gut microbiota.
}

Investigation of $\mathrm{B}_{12}$ Metabolism by Using a Cobalamin Activity-Based Probe

Chemical activity-based probes facilitate identification and measurement of protein activity from any biological sample. Rosnow et al. (e00955-18) used a cobalamin activity-based probe $\left(B_{12}-A B P\right)$ to demonstrate that $B_{12}$-auxotrophic bacteria and archaea can be cultured on the $B_{12}-A B P$, and they present results showing how the probe transports into cells and interacts with protein, RNA, and DNA. Their results suggest that all investigated interactions for enzyme activity, transport, and translational and transcriptional regulation show that the $B_{12}-A B P$ behaves physiologically like vitamin $B_{12}$, allowing archaea and bacteria to grow to physiologically relevant concentrations.

\section{A Metabolic Shift To Improve the Robustness of Lactococcus lactis}

The species Lactococcus lactis is used as a starter for producing lactic acid. However, this acidification has a deleterious effect on cell survival. In contrast, when L. lactis undergoes respiration metabolism, the cell produces much less lactic acid, extending its multiplication and long-term survival capacities once it reaches the stationary phase. Under respiration growth conditions, the cell displays a strong metabolic change, with high-level production of acetate and acetoin at the expense of lactate. Cesselin et al. (e01005-18) show that acetate production allows an increase in biomass yield while acetoin production protects the cell against acidification effects.

\section{Novel Strategy Broadens the R-Tailocin Killing Spectrum in Pseudomonas chlororaphis}

The gene clusters of R-tailocins (bacteriocins resembling contractile bacteriophage tails) typically encode one receptor-binding tail fiber protein per R-tailocin, which determines target specificity. Dorosky et al. (e01230-18) identified three distinct tail fiber-encoding genes within the second of two R-tailocin modules in the R-tailocin gene cluster of Pseudomonas chlororaphis 30-84. This study confirmed that P. chlororaphis 30-84 produces not only two distinct R-tailocins but three different types of the second, each having different tail fibers and killing specificities. This diversity in R-tailocin particles is a mechanism for achieving broader killing activity and greater competitive fitness within rhizosphere microbial communities. 
Streptococcus suis Fimbria-Like Protein SssP1 Contributes to Bacterial Virulence

Streptococcus suis is an important zoonotic pathogen responsible for significant economic losses in the pig industry and public health problems. Zhang et al. (e0138518) demonstrated that SssP1, a novel fimbria-like protein transported by the SecY2/A2 system, contributes to the pathogenicity of $S$. suis. The mechanisms by which $S$. suis constructs these fimbria-like structures on the cell surface and adheres to host cells were also elucidated. These findings provide new insights into $S$. suis pathogenesis as well as the prevention and treatment of its resulting disease. 